\title{
Look, no hands: A perceptual task shows that number magnitude induces shifts of attention
}

\author{
Michael E.R. Nicholls* \\ Andrea M. Loftus* \\ Wim Gevers ${ }^{\#}$ \\ *Department of Psychology \\ University of Melbourne

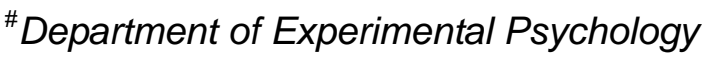 \\ Ghent University
}

Running head: Number magnitude \& attention

Correspondence should be sent to:

Associate Professor Mike Nicholls

Department of Psychology

University of Melbourne

Parkville

VIC 3010

AUSTRALIA

Ph: $\quad 61383444299$

Fax: $\quad 61393476618$

E-Mail: mike.nicholls@unimelb.edu.au 


\section{Abstract}

The mental representation of numbers along a left-to-right oriented line affects spatial cognition, facilitating responses in the ipsilateral hemispace (the Spatial-Numerical Association of Response Codes (SNARC) effect). We investigated whether the number/space association is the result of an attentional shift or response selection. Previous research has often introduced covert left/right response cues by presenting targets to the left or right. The current study avoided left/right cues by requiring forced-choice upper/lower luminance discriminations to two mirror-reversed luminance gradients (the greyscales task). The greyscales stimuli were overlayed with strings of: (a) low numbers, (b) high numbers and (c) non-numerical characters. In Expt. 1, 20 dextrals judged the number's magnitude and then indicated whether the upper/lower greyscale was darker. Results showed leftward and rightward attentional biases for low and high numbers, respectively. Demands to process numbers along a left/right line were made less explicit in Expt $2(N=18$ dextrals) using: (a) a parity judgement and (b) arbitrary linguistic labels for top/bottom. Once again, a spatial congruency effect was observed. Because the response (up/down) was orthogonal to the dimension of interest (left/right), the effect of number cannot be attributed to late-stage response congruencies. This study required un-speeded responses to stimuli presented in free-vision whereas other experiments used speeded responses. Understanding the time course of number-space effects may therefore be important to the debate associated with response selection 
While numbers are conventionally considered to symbolise a mathematical set or object, they can have properties that affect spatial cognition. These number-space properties are clearly exemplified by the Spatial-Numerical Association of Response Codes (SNARC) effect. In a classic demonstration of this effect, Dehaene, Bossini and Giraux (1993) presented numbers between zero and nine and asked participants to make parity judgements (odd vs even) using the left and right hands. Despite the fact that the magnitude of the number was irrelevant to the task, left and right-hand responses were faster to lower (e.g. 1 or 2) or higher numbers (e.g. 8 or 9) numbers, respectively.

The SNARC effect is thought to be associated with an internal representation of numbers along a mental number line, which runs in an ascending order from left to right (Dehaene et al., 1993). Thus, small numbers associated with the left side result in faster left-hand responses. Conversely, large numbers associated with the right side result in faster right-hand responses. While there is some consensus concerning the origin of number-space effects, the level at which the mental representation gives rise to the asymmetry is much less certain. One theory suggests that the asymmetry is related to an attentional shift associated with the perception of the stimulus. Another theory suggests that the asymmetry arises later during response selection.

Support for the attentional shift model has been reported by Fischer, Castel, Dodd and Pratt (2003). Digits (1,2, 8 or 9) were presented centrally, and after a SOA lasting between 50 and 1000ms, a target appeared to the left or right of a central cross. Participants made speeded responses to the onset of the targets using a single button press with the preferred hand. Despite the fact that the numbers were unrelated to target location, responses were faster to targets on the left or right when they were preceded by low (1 or 2) or high (8 or 9) numbers, respectively. Fischer et al. (2003) reasoned that this effect could not be attributed to asymmetries in response selection because all trials required the same, simple reaction time response. Ristic, Wright and Kingstone (2006) and Galfano, Rusconi and Umiltá (2006) have both replicated the effect reported by Fischer et al. (2003) and demonstrated that the attentional asymmetry is driven by top-down, non-obligatory shifts in attention. 
Shifts in attention are also implicated in a temporal order task by Casarotti, Michielin, Zorzi and Umiltá (2007). They found that the presentation of relatively low numbers caused events on the left side of a display to appear earlier than on the right side (and vice versa for high numbers). The results demonstrate that numbers cause a shift of attention to the side of space ipsilateral to the number's position on the mental number line. The fact that this shift occurred for vocal responses that have no spatial component suggests that the association occurs without response conflict.

Support for the response selection model has come from a variety of sources. Keus and Schwarz (2005) tested the comparative importance of attentional and response processes by changing the relative position of the stimuli (affecting perception) and the position of the effectors (affecting response selection). Manipulations of the congruency of the stimuli in relation to the mental number line produced no SNARC effect whereas manipulation of the congruency of the effectors produced a typical SNARC effect. Keus and Schwarz (2005) therefore concluded that the SNARC effect occurred during response selection. Psychophysiological research supports this conclusion. For a parity judgement task, Keus, Jenks and Schwarz (2005) found that stimulus-locked ERPs showed a weak effect of SNARC whereas response-locked ERPs showed a strong SNARC effect. The response selection model is also supported by a body of research comparing the SNARC effect to the Simon effect (Gevers, Lammertyn, Notebaert, Verguts \& Fias, 2006; but see, Mapelli, Rusconi and Umiltá, 2003), though Gevers et al. (2006) did note differences in the time course of the two effects.

The attentional shift and response selection models need not be mutually exclusive - in fact, it is possible that both could operate during numerical processing. The question remains, however: Is it possible to observe numerical/spatial congruency effects in the absence of response competition? The data pertaining to this issue is equivocal. Indeed, it is possible that studies which purport to show that numerical/spatial association is independent of responses selection still encourage participants to code 'left' and 'right' covertly. For example, Fischer et al. (2003) presented targets to the left and right to which a speeded response was required. Although the responses were not lateralised, the arrangement of the stimuli may have encouraged a coding of the left and right targets as left and right 
responses, respectively (see, Kennet, Martin, Spence \& Driver, 2001). Similarly, the left/right appearance of temporal stimuli in the study by Casarotti et al. (2007) may have covertly encouraged left/right coding of the stimuli. It should be noted, however, that overt coding of 'left' and 'right' by presenting the stimuli to the left or right of centre, does not necessarily entail a SNARC effect (See Keus \& Schwarz, 2005). In the current study, we used a novel design where the stimuli are symmetrical and the response dimension is orthogonal to the dimension of interest. Therefore, if we observe an effect of number magnitude, it must reflect a lateralised shift in attention

\section{EXPERIMENT 1}

The primary task required a forced-choice, two-alternative luminance discrimination between two mirror-reversed luminance gradients (the greyscales task), which are placed in the centre of the screen, one above the other. The greyscales task is highly sensitive to lateral biases in attention (Nicholls, Bradshaw \& Mattingley, 1999). Patients with left neglect bias their discrimination toward the stimulus with the salient feature on the right (Mattingley, Berberovic, Corben, Slavin, Nicholls \& Bradshaw, 2003). Thus, when asked to determine which stimulus is darker overall, neglect patients will select the stimulus that is dark on the right. In contrast to patients with left neglect, the general population shows a bias toward selecting the stimulus with the salient feature on the left (Nicholls et al., 1999). This leftward bias is thought to reflect the operation of pseudoneglect, an attentional bias that increases the salience of the leftward features of an object (Bowers \& Heilman, 1980, McCourt \& Jewell, 1999; Jewell \& McCourt, 2000). The influence of number magnitude on space was examined by overlaying the greyscales stimuli with three different strings of characters, composed of: (a) low numbers, (b) high numbers and (c) neutral symbols (see Fig. 1). To assure that the number's magnitude was activated, participants made judgements of relative magnitude by stating whether the characters were: (a) lower than five, (b) higher than five or (c) neutral.

In some respects, the greyscales task is analogous to line bisection tasks where the lines were composed of digits (Fischer, 2001) or number-words (Calabria \& Rossetti, 2005). Both studies found 
that, for lines composed of small numbers, bisections were shifted to the left. While the studies do show an effect of number magnitude, the act of physically bisecting the line introduces a motor component which could be oriented to the left or right. Calabria and Rossetti $(2005$, p. 782$)$ therefore noted: "The present data cannot explain whether numbers affect performance at a sensory, representational or motor level because any of these three components may be affected by our simple task". The current study is ideally suited to addressing this issue because the task required participants to state whether the upper or lower greyscales stimulus was darker overall. The verbal response of 'upper' and 'lower' has no motor or representational (though, see Expt. 2) component that is directly related to the dimension of interest (left/right). As a result, there can be no congruency effect related to the representation of the mental number line and the response. If the association between numerical magnitude and space is driven by shifts of attention that are independent of response selection, overlays containing low and high numbers should cause leftward and rightward biases for the greyscales task, respectively. In line with the pseudoneglect literature, which proposes a consistent, but subtle, leftward bias (McCourt, 2001), a moderate leftward bias was anticipated for the neutral stimuli.

\section{Method}

Participants. Twenty students $(\mathrm{f}=16, \mathrm{~m}=4)$ aged between 18 and 27 years (mean=22.14 years) from the University of Melbourne participated in this experiment. The participants were right-handed (Oldfield, 1971) and had normal or corrected-to-normal vision.

Apparatus. Stimuli were presented on a $280 \mathrm{~mm} \times 375 \mathrm{~mm}$ CRT monitor. A chin rest maintained head position at 500mm from the monitor. An intercom system positioned in front of the participant allowed the experimenter to hear verbal responses clearly.

Stimuli. For each trial, participants viewed two greyscales stimuli simultaneously. Each greyscales stimulus was defined by a thin black rectangle $\left(0.6 \mathrm{~mm}\left(0.1^{\circ}\right)\right.$ thick) and displayed against a white background. The stimuli were either $85 \mathrm{~mm}\left(9.6^{\circ}\right)$ or $102 \mathrm{~mm}\left(11.5^{\circ}\right)$ long and $18 \mathrm{~mm}\left(2.1^{\circ}\right)$ high. The horizontal midline of each stimulus pair was aligned with the screen's 
centre, while the vertical midline of the upper and lower stimuli were placed $30 \mathrm{~mm}\left(3.4^{\circ}\right)$ above and below the centre. Each stimulus changed in luminance from one end to the other by a linear adjustment of the ratio of white to black pixels (for precise details of the construction of the greyscales stimuli, see: Nicholls et al., 1999). Despite the fact that the stimuli were left/right mirror reversals of each other, they were equiluminant at a global level.

\section{INSERT FIGURE 1 ABOUT HERE}

Procedure. The factorial combinations of stimulus length (long, short), polarity (upper stimulus dark on left, lower stimulus dark on right \& vice versa) and overlay $(1,2,8,9$, \#, \&) were repeated 10 times, resulting in 240 trials. The trials were split into three blocks, within which the factorial combinations of stimulus length, polarity and overlay changed from trial to trial in a pseudo-randomized order. Participants were seated centrally in relation to the monitor. Each stimulus pair was presented for three seconds. There was a two second inter-trial interval, during which participants fixated a central cross. Participants performed two tasks. First, participants identified the category of the overlay by stating 'low' $(1,2)$, 'high' $(8,9)$ or 'neutral' $(\&, \#)$. Second, participants indicated which stimulus within the pair was overall darker by stating 'top' or 'bottom'. Participants completed the tasks in this order and made their response during the two-second inter-trial interval. Prior to commencing the experiment, participants completed 10 practice trials.

\section{Results \& Discussion}

For each trial, participants selected the stimulus that was darker on the left or the right. These were classified as 'left' and 'right' responses, respectively. Response bias was calculated by subtracting the number of left responses from the number of right responses and converting the result into a percentage of the total number of trials. Response bias scores therefore ranged from -100 (leftward bias) to +100 (rightward bias).

The data were analysed with an ANOVA with overlay (low, high or neutral) as a withinparticipant factor. Effect sizes are expressed as partial eta-squared values. Figure 2 shows a 
main effect of overlay $\left(F(2,38)=18.83, p<.001, \eta^{2}=.498\right)$. Post-hoc contrasts revealed that the bias for the high overlay was significantly to the right of the neutral $(t(19)=3.41, p<.005)$ and low $(\mathrm{t}(19)=4.85, \mathrm{p}<.001)$ overlays. The bias for the low overlay was significantly leftwards of the bias for neutral overlay $(\mathrm{t}(19)=4.48, \mathrm{p}<.001)$.

\section{INSERT FIGURE 2 ABOUT HERE}

In line with our predictions, there was a strong effect of numerical overlay whereby a leftward bias for small numbers reversed to a rightward bias for large numbers. The data therefore demonstrate that spatial congruency effects can arise in the absence of any motor activity directed to the left or right. While the neutral overlays produced an intermediate level of bias, the leftward bias failed to reach statistical significance. Although the pseudoneglect literature suggests that a leftward bias should emerge in this condition (Nicholls et al., 1999), the literature also demonstrates that pseudoneglect effects are variable and affected by small task-related factors (McCourt, 2001).

\section{EXPERIMENT 2}

The first experiment deliberately encouraged participants to engage the mental number line by requiring a judgement of relative order. Within the context of alphabetical stimuli, Gevers, Reynvoet and Fias (2003) found that left/right spatial coding was stronger when the task required judgements of order and weaker when order was irrelevant. Nevertheless, the majority of research into the SNARC effect has elicited spatial coding effects with tasks such as parity judgements, where the judgement is orthogonal to the number's position in the mental number line (e.g. Dehaene et al., 1993). Techniques, such as this, demonstrate that the mental number line is activated automatically in the absence of any cues. In the view of this, the second experiment removed any explicit cues to process magnitude by introducing a parity judgment, requiring a response of 'odd' to digits of 1 and 9 and 'even' to 2 and 8 . If the spatial coding effect is engaged automatically, we would expect a similar effect of overlay to that observed in the first study. Bearing in mind the data collected by Gevers et al. (2003) it also seems reasonable to expect that the spatial congruency effect may be weaker for this experiment compared to the first experiment. By removing discriminations of magnitude, we were also able to remove any 
confusion between the response to the overlay ('higher' or 'lower' than five) and the response to the greyscales stimuli ('upper' or 'lower' stimulus darker).

In the first experiment, participants indicated their decision by responding 'top' or 'bottom'. It was argued that the top/bottom response dimension was unrelated to the dimension of interest (left/right). There is a body of research, however, which shows stimulus-response compatibility effects between top/right and bottom/left (Bauer \& Miller, 1982; Proctor \& Cho, 2006). In addition, research by Ito and Hatta (2004) has shown a vertical SNARC effect whereby low and high numbers are placed towards the bottom and top of space, respectively (also see, Schwarz \& Keus, 2004). It is therefore possible that verbal response of 'top' and 'bottom' primed mental representations of right and left. Furthermore, responses of 'top' and 'bottom' may have primed a vertical representation of the mental number line. To remove any response priming effects, red or blue boxes flanked the upper and lower greyscales stimuli. Participants selected the stimulus that looked darker overall by saying 'red' or 'blue'.

\section{Method}

Participants. Eighteen students $(\mathrm{f}=11, \mathrm{~m}=7)$ aged between 18 and 28 years (mean=23.1 years) from the University of Melbourne participated in this experiment. All other characteristics of the participants were the same as those described in the first experiment. None of the participants took part in the first experiment.

Apparatus \& stimuli. The apparatus and stimuli were the same as that used in the first experiment, with the following exception. On each trial, four $18 \mathrm{~mm}\left(2.1^{\circ}\right)$ squares were presented, with two falling $90 \mathrm{~mm}\left(10.2^{\circ}\right)$ to the left of centre and two falling $90 \mathrm{~mm}$ to the right. The squares were aligned with the vertical centre of each greyscales stimulus, so that two flanked the upper greyscales stimulus and two flanked the lower stimulus. To avoid interference effects between the squares and the greyscales stimuli, the medial edge of the squares was never closer than $39 \mathrm{~mm}\left(4.5^{\circ}\right)$ from the lateral edge of the greyscale stimuli. The squares flanking the upper or lower stimuli were both either red or blue. If the upper squares were blue, the lower squares were red (and vice versa). 
Procedure. The procedure was essentially the same as the first experiment, with the following exceptions. First, participants were asked to make parity judgments for the numerical stimuli, responding 'odd' for 1 and 9 and 'even' for 2 and 8. Like experiment 1, participants responded 'neutral' for the \# and \& characters. Participants then indicated whether the upper or lower stimulus was darker overall. To indicate their choice, participants responded by naming the colour (red or blue) of the squares that flanked the stimulus. For half of the trials the upper stimulus was flanked by blue squares and the lower stimulus by red squares. The arrangement was reversed for the other half of trials.

\section{Results \& Discussion}

Figure 2 shows a main effect of overlay $\left(F(2,34)=6.85, p<.005, \eta^{2}=.287\right)$. Contrasts between the overlays revealed that the bias for the high overlay was significantly to the right of the biases for the neutral $(t(17)=2.48, p<.05)$ and the low $(t(17)=2.76, p<.05)$ overlays. The leftward bias was stronger for the low compared to the neutral overlay, but this difference just failed to reach statistical significance $(t(17)=1.88, p=.08)$. To compare the results between experiment 1 and 2, the data were combined and analysed with an ANOVA with experiment (1 vs 2$)$ as a between participants factor. The effect of overlay remained significant $(F(2,72)=22.04$, $\left.p<.001, \eta^{2}=.380\right)$. The overall leftward bias was slightly stronger in the second experiment compared to the first, resulting in a main of experiment that approached significance $\left(F(1,36)=3.78, p=.06, \eta^{2}=.095\right)$. Importantly, there was no interaction between experiment and overlay $(F(2,72)=1.01, n s)$.

Like experiment 1, there was a large difference in the biases caused by the low and high overlays. For low overlays, there was a leftward bias, which reversed to a non-significant rightward bias for the high overlays. The effect of number magnitude is compatible with a spatial congruency effect where low numbers are located to the left and high numbers are located to the right (Dehaene et al., 1993). Unlike experiment 1, there was no outright rightward bias for the high overlay condition. The lack of rightward bias can be explained with reference to the neutral overlay condition, which produced a leftward bias. This bias is consistent with the effect of pseudoneglect, where an attentional bias 
towards the left hemispace increases the salience of features located on the left (Nicholls et al., 1999). Given that pseudoneglect sets the baseline to the left of centre, it is perhaps not surprising that the rightward shift for high numbers was not significantly to the right of centre. The strong leftward bias for the neutral condition also resulted in a comparatively small leftward shift for low overlays relative to the neutral condition - perhaps reflecting a ceiling effect.

The data demonstrate that the spatial congruency effect observed in the first experiment generalises to judgements of parity. Given that parity decisions do not require judgments about a number's relative position on the number line, the data demonstrate that the number line is engaged automatically. The results are therefore consistent with other studies showing spatial congruency effects when the discrimination is unrelated to the left/right dimension of the number line (Dehaene et al., 1993). That said, the effect of high and low overlays does appear to be weaker than the first experiment. The difference in effect size between the experiments is consistent with the data reported by Gevers et al. (2003), who also reported weaker spatial effects when the task did not require magnitude judgements.

Experiment 1 used responses of 'top' and 'bottom', which may not be neutral in relation to the dimension of left and right (Bauer \& Miller, 1982; Proctor \& Cho, 2006) or to the mental number line (Ito \& Hatta, 2004). Explicit verbal coding of up and down was avoided in Experiment 2 using arbitrary response of 'red' and 'blue'. Despite this change, clear spatial congruency effects were observed demonstrating that the effect is not due to verbal coding and congruency effects.

\section{GENERAL DISCUSSION}

The two experiments described in the present study eliminated congruency effects in response mapping by requiring participants to make verbal responses which were orthogonal to the dimension of interest (right vs left). By eliminating left/right coding of the stimuli, we have avoided any covert processing of stimuli in terms of action-related planning (Hommel, Müsseler, Aschersleben \& Prinz, 2001). The results therefore demonstrate that numbers affect spatial attention in the absence of conflicts associated with response selection. Research by Galfano et al (2006) and Ristic et al. (2006) 
suggests that the attention shifts associated with number magnitude are likely to be endogenous in nature and driven by non-obligatory top-down processes.

In addition to the debate associated with attentional shifts vs response selection, the present data also provide interesting information concerning the time scale of number-space effects. SNARC effects have traditionally been investigated using speeded discriminations to centrally presented stimuli (Dehaene et al., 1993), yielding reaction times in the order of 500ms (e.g Keus et al., 2005; Mapelli et al., 2003). The discriminations would have therefore required participants to assimilate the information quickly and to make a rapid response. The current study presented the stimuli for three seconds and did not require a speeded response. The data therefore demonstrate that number-space effects are not restricted to transitory shifts of attention, but also occur for more sustained periods. Understanding the time course of number-space effects may be important to the debate associated with response selection. It is interesting to note that Fischer et al. (2003) and Casarotti et al. (2007) observed a number-space effect when the digit was presented at least $400 \mathrm{~ms}$ prior to the target. This suggests that, in order for early-stage, perceptual processes to have an effect, time needs to be allowed for an attentional bias to develop. Direct evidence for this proposition comes from Stoianov, Kramer, Umiltá and Zorzi (in press) who found an interaction between spatial and number cues only when sufficient time was allowed for an expectancy to develop for the number cue. For studies that require a speeded response to the number itself (e.g. Keus et al., 2005; Mapelli et al., 2003), there may not be sufficient time for a sustained expectancy to develop. Speeded responses may therefore encourage responserelated congruencies and may be less voluntary. In conclusion, although we have demonstrated that a number-space effect emerges at an early, sensory-stage, it is likely that tasks that decrease number processing time and accentuate response-related incongruencies will lead to locus at a later, motorstage. 


\section{References}

Bauer, D.W., \& Miller, J. (1982). Stimulus-response compatibility and the motor system. Quarterly Journal of Experimental Psychology, 34A, 367-380.

Bowers D., \& Heilman, K.M. (1980). Pseudoneglect: Effects of hemispace on a tactile line bisection task. Neuropsychologia, 18, 491-498.

Calabria, M., \& Rossetti, Y. (2005). Interference between number processing and line bisection: a methodology. Neuropsychologia, 43, 779-783.

Casarotti, M., Michielin, M., Zorzi, M., \& Umiltá, C. (2007). Temporal order judgements reveal how number magnitude affects visuospatial attention. Cognition, 102, 101-117.

Dehaene, S., Bossini, S., \& Giraux, P. (1993) The mental representation of parity and number magnitude. Journal of Experimental Psychology: General, 122, 371-396.

Fischer, M.H. (2001). Number processing induces spatial performance biases. Neurology, 57, 822-826.

Fischer, M.H., Castel, A.D., Dodd, M.D., \& Pratt, J. (2003). Perceiving numbers causes shifts of attention. Nature Neuroscience, 6, 555-556.

Galfano, G., Rusconi, E., \& Umiltá, C. (2006). Number magnitudes orients attention, but not against one's will. Psychonomic Bulletin \& Review, 13, 869-874.

Gevers, W., Lammertyn, J., Notebaert, W., Verguts, T., \& Fias, W. (2006). Automatic response activation of implicit spatial information: Evidence from the SNARC effect. Acta Psychologica, $122,221-233$.

Gevers, W., Reynvoet, B., \& Fias, W. (2003). The mental representation of sequences is spatially organized. Cognition, 87, 87-95.

Hommel, B., Müsseler, J., Aschersleben, G., \& Prinz, W. (2001). A theory of event coding (TEC): A framework for perception and action planning. Behavioral and Brain Sciences, 24, 849937. 
Ito, Y., \& Hatta, T. (2004). Spatial structure of quantitative representation of numbers: Evidence from the SNARC effect. Memory \& Cognition, 32, 662-673.

Jewell, G. \& McCourt, M.E. (2000). Pseudoneglect: A review and meta-analysis of performance factors in line bisection tasks. Neuropsychologia, 38, 93-110.

Kennett, S., Martin, E., Spence, C. and Driver, J. (2001). Tactile-visual links in exogenous spatial attention under different postures: Convergent evidence from psychophysics and ERPs. Journal of Cognitive Neuroscience, 13, 462-478.

Keus, I.M., Jenks, K.M., \& Schwarz, W. (2005). Psychophysical evidence that the SNARC effect has its functional locus in the response selection stage. Cognitive Brain Research, 24, 48-56.

Keus, I.M., \& Schwarz, W. (2005). Searching for the functional locus of the SNARC effect: evidence for a response-related origin. Memory \& Cognition, 33, 681-695.

Mapelli, D., Rusconi, E., \& Umiltá (2003). The SNARC effect: an instance of the Simon effect? Cognition, 88, B1-B10.

Mattingley, J.B., Berberovic, N., Corben, L., Slavin, M.J., Nicholls, M.E.R., \& Bradshaw, J.L. (2003). The greyscales task: a perceptual measure of attentional bias following right hemisphere damage. Neuropsychologia, 42, 387-394.

McCourt, M.E. (2001). Performance consistency of normal observers in forced-choice tachistoscopic visual line bisection. Neuropsychologia, 39, 1065-1076.

McCourt, M.E., \& Jewell, G. (1999). Visuospatial attention in line bisection: Stimulus modulation of pseudoneglect. Neuropsychologia, 37, 843-855.

Nicholls, M.E.R., Bradshaw, J.L., \& Mattingley, J.B. (1999). Free-viewing perceptual asymmetries for the judgement of shade, numerosity and size. Neuropsychologia, 37, 307-314.

Oldfield, R.C. (1971). The assessment of handedness: the Edinburgh Inventory. Neuropsychologia, 9, 97-133.

Proctor, R.W., \& Cho. Y.S. (2006). Polarity correspondence: A general principle for performance of speeded binary classification tasks. Psychological Bulletin, 132, 416-442. 
Ristic, J., Wright, A., \& Kingstone, A. (2006). The number line reflects top-down control. Psychonomic Bulletin \& Review, 13, 862-868.

Schwarz, W., \& Keus, I.M. (2004). Moving the eyes along the mental number line: Comparing SNARC effects with saccadic and manual responses. Perception \& Psychophysics. 66, 651-664.

Stoianov, I., Kramer, P., Umiltá, C. \& Zorzi, M. (in press). Visuospatial priming of the mental number line. Cognition. 


\section{Figure captions}

Fig. 1. Example of the greyscales stimuli. Each stimulus pair contained one of six overlays, comprised of two low numbers $(1 \& 2)$, two high numbers $(8 \& 9)$ and two non-numerical symbols ( $\&$ and \#). The symbols were drawn using a size 36 Arial font and were defined by a thin grey line with a transparent inner area. Each overlay contained eight (short stimuli) or ten (long stimuli) repetitions of the same symbol. Overlays for the top and bottom bars were identical.

Fig. 2. Mean percentage response bias (with \pm SE bars) for the three different overlay conditions in Experiment 1 and 2. Negative and positive response bias scores indicate leftward and rightward response biases, respectively. T-tests were conducted comparing each of the six conditions against zero (no bias). Conditions that were significantly different from zero are indicated by an asterisk. 


\section{Acknowledgements}

We would like to thank Mark McCourt, Silke Göbel and an anonymous review for their helpful comments on an earlier version of this manuscript. 
Figure 2

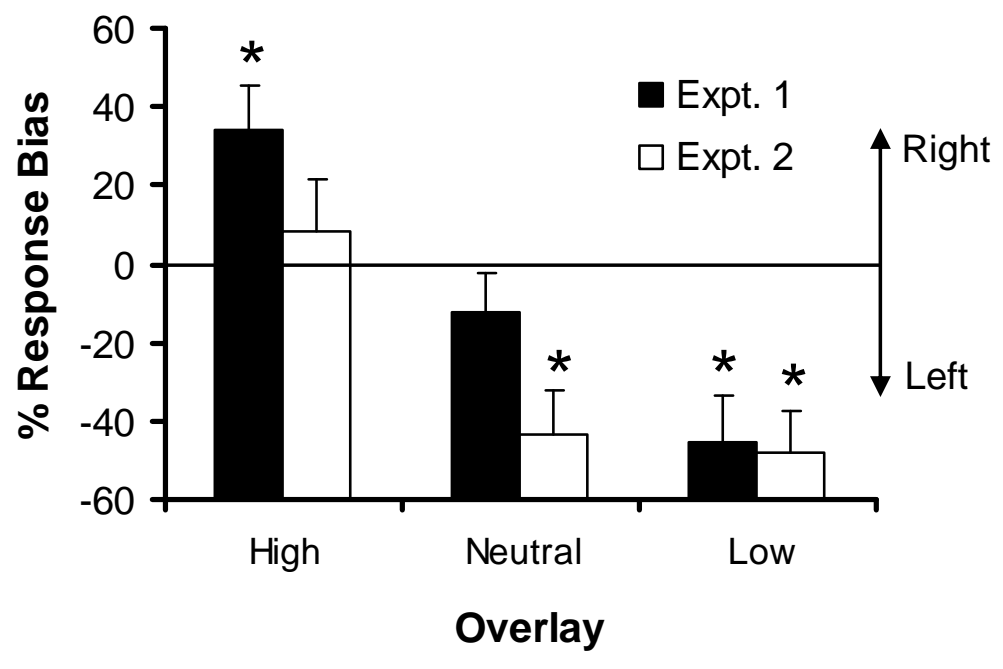


Figure 1.

(a)
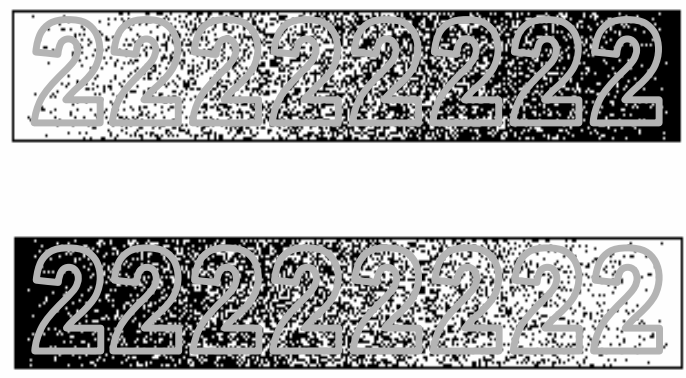

(b)


(c)
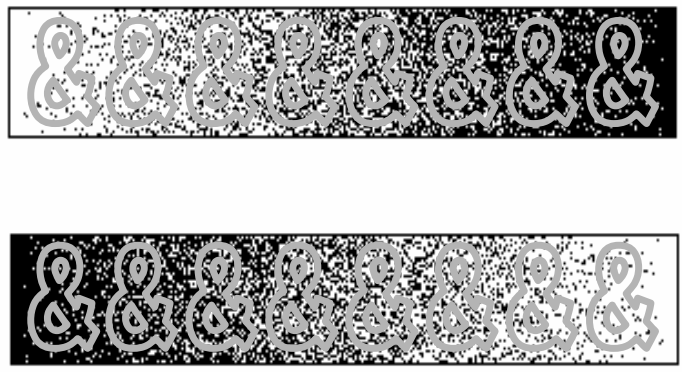

Polarity 2
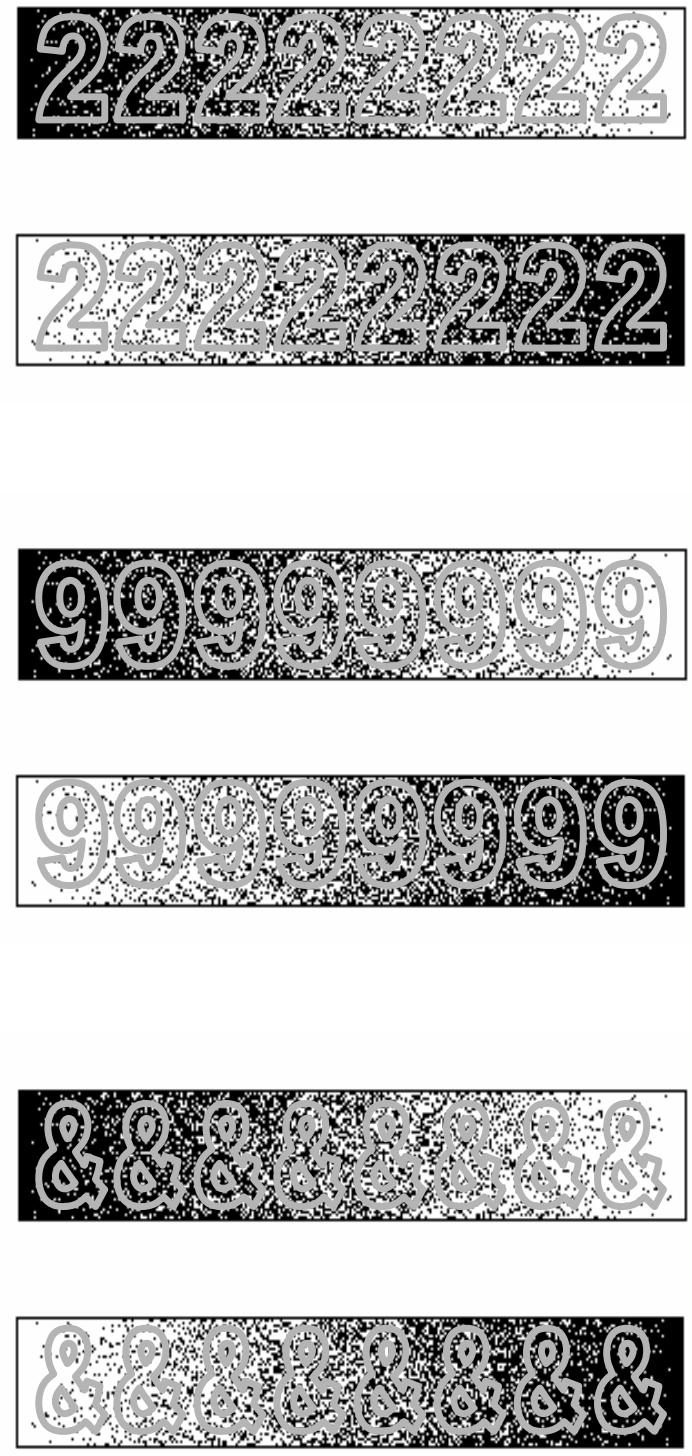